\title{
BEM in free vibration analysis of elastic shells coupled with liquid sloshing
}

\author{
K. Degtiariov, V. Gnitko, V. Naumenko \& E. Strelnikova \\ A. N. Podgorny Institute for Mechanical Engineering Problems \\ of the National Academy of Sciences of Ukraine, Ukraine
}

\begin{abstract}
This paper deals with the fluid-structure interaction analysis of a shell partially filled with a liquid. The shell is considered to be thin and the Kirghoff-Lave linear theory hypotheses are applied. The liquid is ideal and incompressible. The problem of analysing the dynamics of shells of revolution partially filled with an ideal incompressible liquid was reduced to solving the system of singular integral equations. The solution was obtained by using a coupled BEM and FEM in-house solver. The tank structure is modelled by the FEM and the liquid sloshing in the fluid domain is described by the BEM. The shell vibrations coupled with liquid sloshing under the force of gravity were considered. The shell and sloshing modes were analysed simultaneously. The free vibration analysis of the elastic cylindrical shell was carried out using the proposed techniques.
\end{abstract}

Keywords: fluid-structure interaction, liquid sloshing, free vibrations, boundary and finite element methods, systems of singular integral equations.

\section{Introduction}

Sloshing is the low frequency oscillations of the free surface of a liquid in a partially filled container. The dynamic response of structures containing the liquid can be significantly influenced by these oscillations and their interaction with the sloshing liquid may lead to instabilities in different engineering areas such as aerospace and chemical industry, power machine building, wind power engineering, transport, etc. Usually liquid storage tanks are filled with oil, flammable or toxic liquids. Destruction of these tanks by seismic action or shockwaves from a nearby explosion can lead to environmental catastrophe. So 
seismic design of liquid storage tanks requires the knowledge of sloshing frequencies and the hydrodynamic pressure on the walls [1]. Complex experimental investigation of loading processes is difficult and sometimes impossible for various reasons. Hence mathematical modeling of physical processes with the help of advanced computer techniques is a basic approach for these problems. Numerical methods are especially useful when the geometry of the container is complicated and the sloshing in the container cannot be analytically investigated. Various approaches have been proposed to research the fluid-structure interaction problems, including the finite difference methods [2], the finite element methods [3], the boundary element methods (BEM) [5-10]. The research findings are summarized in [11]. The dynamic analysis of shell structures is usually accomplished by usage of the finite element programs. However such 3D finite element analysis including the contained fluid is complex and extremely time consuming. In [7-9] authors offer the approach based on the boundary element method to the problem of free vibrations of fluidfilled shell of revolution as well as to the problem of liquid sloshing in rigid tanks.

In this paper the free vibration analysis of an elastic cylindrical shell coupled with the liquid sloshing is carried out. Here we use combination of reduced finite and boundary element methods. The analysis consists of several stages, each represents a separate task. The frequencies and modes of the empty shell vibrations are defined in the first stage. The displacement vector, that is the solution of the coupled problem, is sought as a linear combination of natural modes of an empty shell. So we define the frequencies and free vibrations modes of the fluid-filled elastic shell without including the force of gravity. Then we obtain the frequencies and free vibrations modes of the liquid in the rigid shell under the force of gravity. Two latter problems are solved using the reduced BEM. Then we come to the problem of coupled analysis of liquid sloshing and structural vibrations.

\section{Problem statement}

Let us consider the coupled problem for the shell of revolution interacting with a liquid (Figure 1). We indicate the wetted part of the shell surface through $S_{1}$ and the free surface of a liquid as $S_{0}$. Suppose that the shell geometry is defined with respect to a global Cartesian coordinate system $x, y, z$ and the free surface located in $x \mathrm{O} y$ plane in a state of rest.

The main objective of this paper is to find out and evaluate modes and frequencies of free vibrations of an elastic shell of revolution coupled with liquid sloshing. In this study the contained liquid is assumed to be inviscid and incompressible one and its flow induced by vibrations of the shell is irrotational.

Under these suppositions, there exists a velocity potential $\Phi$ satisfying the Laplace equation. The equations of motion of the two media (the shell, $S_{1}$, and the fluid with free surface, $S_{0}$, (see Figure 1)) can be written in the following form:

$$
\mathbf{L}(\mathbf{U})+\mathbf{M}(\ddot{\mathbf{U}})=\mathbf{P}
$$




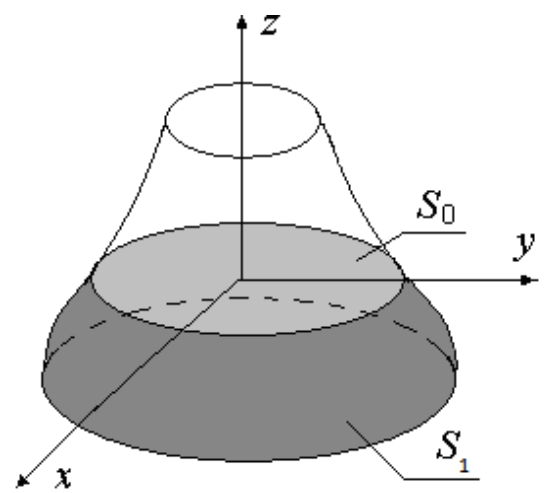

Figure 1: A shell of revolution partially filled with a liquid.

where $\mathbf{U}$ is the vector-function of displacements, $\mathbf{P}$ is the fluid pressure on a moistened surface of the shell, and $\mathbf{L}$ and $\mathbf{M}$ are the operators of elastic and mass forces.

Let us consider the right-hand side of equation (1). Notice that the vector $\mathbf{P}$ points in the normal direction to the shell wall because an ideal fluid produces only a normal pressure on a moistened body. We will denote $|\mathbf{P}|=p$. Assuming that the natural velocity of the fluid is zero, the value $p$, according to the CauchyLagrange integral, can be represented as follows

$$
p=-\rho_{l}\left(\Phi_{t}^{\prime}+g z\right)+p_{0},
$$

where $\Phi$ is the velocity potential, $g$ is the free fall gravity acceleration, $z$ is the vertical coordinate of a point in the liquid, $p_{0}$ is the atmospheric pressure and $\rho_{l}$ is the fluid density. To obtain the boundary equations on the free surface we have formulated dynamic and kinematics boundary conditions. The dynamic boundary condition consists in equality of the liquid pressure on the free surface to atmospheric one. The kinematics boundary condition requires that liquid particles of the free surface remain on it all the time of subsequent motion. So we have

$$
\left.\frac{\partial \Phi}{\partial n}\right|_{S_{0}}=\frac{\partial \zeta}{\partial t} ; \quad p-\left.p_{0}\right|_{S_{0}}=0,
$$

where an unknown function $\zeta=\zeta(t, x, y, z)$ describes the form and location of the free surface. Thus, we obtain the following boundary value problem to define the velocity potential $\Phi$ :

$$
\nabla^{2} \Phi=0,\left.\frac{\partial \Phi}{\partial \mathbf{n}}\right|_{S_{1}}=\frac{\partial w}{\partial t},\left.\frac{\partial \Phi}{\partial \mathbf{n}}\right|_{S_{0}}=\frac{\partial \zeta}{\partial t} ; \quad p-\left.p_{0}\right|_{S_{0}}=0 .
$$

Here $w$ indicates the normal component of the shell deflection, $\mathbf{n}$ is an external unit normal to the shell wetted surface. So we reduce the problem under consideration to the following system of differential equations: 


$$
\mathbf{L}(\mathbf{U})+\mathbf{M}(\ddot{\mathbf{U}})=p \mathbf{n} ; p=-\rho_{l}\left(\frac{\partial \Phi}{\partial t}+g z\right)+p_{0} ; \Delta \Phi=0
$$

with the next set of boundary conditions relative to $\Phi$

$$
\left.\frac{\partial \Phi}{\partial \mathbf{n}}\right|_{S_{1}}=\frac{\partial w}{\partial t},\left.\frac{\partial \Phi}{\partial \mathbf{n}}\right|_{S_{0}}=\frac{\partial \zeta}{\partial t}, \frac{\partial \Phi}{\partial t}+\left.g z\right|_{s_{0}}=0
$$

and fixation conditions of the shell relative to $\mathbf{U}$.

To define coupled modes of harmonic vibrations we represent the vector $\mathbf{U}$ in the form $\mathbf{U}=\mathbf{u} \exp (i \omega t)$, where $\omega$ is an own frequency and $\mathbf{u}$ is a mode of vibration of the considered shell with a fluid.

\section{The mode superposition method for coupled dynamic problems}

We will seek modes of the fluid-filled shell vibrations in the form

$$
\mathbf{u}=\sum_{k=1}^{N} c_{k} \mathbf{u}_{k},
$$

where $c_{k}$ are unknown coefficients and $\mathbf{u}_{k}$ are the normal modes of vibrations of the empty shell. In other words, a mode of vibration of the shell filled with a fluid is determined as a linear combination of normal modes of its vibration without liquid.

Let us note that the following relationships are valid

$$
\mathbf{L}\left(\mathbf{u}_{k}\right)=\Omega_{k}^{2} \mathbf{M}\left(\mathbf{u}_{k}\right), \quad\left(\mathbf{M}\left(\mathbf{u}_{k}\right), \mathbf{u}_{j}\right)=\delta_{k j} .
$$

Hence

$$
\left(\mathbf{L}\left(\mathbf{u}_{k}\right), \mathbf{u}_{j}\right)=\Omega_{k}^{2} \delta_{k j},
$$

where $\Omega_{k}$ is the $k$-th frequency of the empty shell vibrations. The above relations (3), (4) show that the abovementioned shell's modes of vibration must be orthonormalized with respect to the mass matrix.

Let us seek $\Phi$ as a sum of two potentials $\Phi=\Phi_{1}+\Phi_{2}$ as it was proposed in [9] Represent the potential $\Phi_{1}$ as the following series expansion:

$$
\Phi_{1}=\sum_{k=1}^{N} \dot{c}_{k} \phi_{1 k}
$$

Here time-dependant coefficients $c_{k}$ are defined in equation (2).

To determine $\varphi_{1 k}$ we have the following boundary value problems:

$$
\Delta \phi_{1 k}=0,\left.\frac{\partial \phi_{1 k}}{\partial \mathbf{n}}\right|_{S_{1}}=w_{k},\left.\phi_{1 k}\right|_{S_{0}}=0 .
$$

Note that the solution of boundary value problem (6) was obtained in [9].

To determine the potential $\Phi_{2}$ we have to solve the problem of fluid vibrations in the rigid vessel taking into account the gravitational force. It leads to the following representation of the potential $\Phi_{2}$ :

$$
\Phi_{2}=\sum_{k=1}^{M} \dot{d}_{k} \phi_{2 k},
$$


where functions $\varphi_{2 k}$ are natural modes of the liquid sloshing in the rigid tank. To obtain these modes we have solved the following sequence of boundary value problems:

$$
\begin{gathered}
\Delta \psi_{2 k}=0 ;\left.\frac{\partial \psi_{2 k}}{\partial \mathbf{n}}\right|_{S_{1}}=0 ; \\
\left.\frac{\partial \psi_{2 k}}{\partial \mathbf{n}}\right|_{S_{0}}=\frac{\partial \varsigma}{\partial t} ; \quad \frac{\partial \psi_{2 k}}{\partial t}+g \zeta=0 .
\end{gathered}
$$

We have differentiated the second equation in relationship (9) with respect to $t$ and substituted there the expression for $\varsigma_{t}^{\prime}$ from the first one of (9). Suppose hereinafter that $\psi_{2 k}(t, x, y, z)=e^{i \chi_{k} t} \phi_{2 k}(x, y, z)$. Next, we obtain the sequence of eigenvalue problems with following conditions on the free surface for each $\varphi_{2 k}$ :

$$
\frac{\partial \phi_{2 k}}{\partial n}=\frac{\chi_{k}^{2}}{g} \phi_{2 k} \text {. }
$$

The effective numerical procedure for solution of these eigenvalue problems using the boundary element method was introduced in [6].

Finally, we obtain the following expression for the potential $\Phi$ :

$$
\Phi=\sum_{k=1}^{N} \dot{c}_{k} \phi_{1 k}+\sum_{k=1}^{M} \dot{d}_{k} \phi_{2 k} .
$$

It follows from equation (11) that function $\zeta$ can be written as

$$
\zeta=\sum_{k=1}^{N} c_{k} \frac{\partial \phi_{1 k}}{\partial n}+\sum_{k=1}^{M} d_{k} \frac{\partial \phi_{2 k}}{\partial n} .
$$

So, the total potential $\Phi$ satisfies the Laplace equation and the nonpenetration boundary condition due to validity of relations (6), (8). Noted that $\Phi$ also satisfies the kinematics boundary condition as a result of representation (12). Satisfying the condition $\Phi_{t}^{\prime}+\left.g z\right|_{s_{0}}=0$ on the free surface, one can obtain the next equality

$$
\sum_{k=1}^{N} \ddot{c}_{k} \phi_{1 k}+\sum_{k=1}^{M} \ddot{d}_{k} \phi_{2 k}+g\left(\sum_{k=1}^{N} c_{k} \frac{\partial \phi_{1 k}}{\partial z}+\sum_{k=1}^{M} d_{k} \frac{\partial \phi_{2 k}}{\partial z}\right)=0 .
$$

When functions $\varphi_{1 k}$ and $\varphi_{2 k}$ are defined, we substitute them in equation (1) and obtain the following relations:

$$
\begin{gathered}
L\left(\sum_{k=1}^{N} c_{k}(t) \mathbf{u}_{k}\right)+M\left(\sum_{k=1}^{N} \ddot{c}_{k}(t) \mathbf{u}_{k}\right)=-\rho_{l}\left(\sum_{k=1}^{N} \ddot{c}_{k}(t) \phi_{1 k}+\sum_{k=1}^{M} \ddot{d}_{k}(t) \phi_{2 k}\right) \\
\sum_{k=1}^{M} \ddot{d}_{k} \phi_{2 k}+g \sum_{k=1}^{N} c_{k} \frac{\partial \phi_{1 k}}{\partial n}+\sum_{k=1}^{M} d_{k} \chi_{k}^{2} \phi_{2 k}=0 .
\end{gathered}
$$

Here we have simplified equation (13) taking into account equations (6), (10). The first equation in (14) is valid on the wetted surface of the shell and the second one - on the free surface of the liquid. Considering the result of dot product of the first equation in (14) by $u_{j}$ and the second one by $\phi_{2 j}$, taking into account relationships (3), (4) and orthogonality of natural modes of fluid 
vibrations in the rigid vessel, we come to the next set of $n+m$ second order differential equations to determine the unknown coefficients $c_{k}(t), d_{k}(t)$ :

$$
\begin{gathered}
c_{j}(t) \Omega_{j}^{2}+\ddot{c}_{j}(t)=-\rho_{l}\left(\sum_{k=1}^{N} \ddot{c}_{k}(t)\left(\phi_{1 k}, w_{j}\right)+\sum_{k=1}^{M} \ddot{d}_{k}(t)\left(\phi_{2 k}, w_{j}\right)\right) \\
\ddot{d}_{j}(t)+g \sum_{k=1}^{N} c_{k}(t)\left(\frac{\partial \phi_{1 k}}{\partial n}, \phi_{2 j}\right)+g \chi_{j}^{2} d_{j}(t)=0 .
\end{gathered}
$$

To define coupled modes of harmonic vibrations we represent the timedependant unknown coefficients as

$$
c_{k}(t)=C_{k} e^{i \omega t} ; \quad d_{k}(t)=D_{k} e^{i \omega t},
$$

where $\omega$ is an own frequency, and $C_{k}, D_{k}$ are unknown constants.

Taking into account equations (16), one can obtain that equations (15) can be expressed as

$$
\begin{gathered}
C_{j} \Omega_{j}^{2}-\omega^{2} C_{j}+\rho_{l}\left(\omega^{2} \sum_{k=1}^{N} C_{k}\left(\phi_{1 k}, w_{j}\right)+\omega^{2} \sum_{k=1}^{M} D_{k}\left(\phi_{2 k}, w_{j}\right)\right)=0, j=\overline{1, N} ; \\
\chi_{l}^{2} D_{l}-\omega^{2} D_{l}+g \sum_{k=1}^{m} C_{k}\left(\frac{\partial \phi_{1 k}}{\partial n}, \phi_{2 l}\right)=0, \quad l=\overline{1, M} .
\end{gathered}
$$

Introducing the following matrixes and vectors

$$
\begin{gathered}
C=\left(C_{k}\right) ; \quad D=\left(D_{k}\right) ; H_{\chi}=\left(\chi_{j}^{2} \delta_{i j}\right) ; H_{\omega}=\left(\Omega_{k}^{2} \delta_{k i}\right) ; \\
A=\left\{a_{j k}\right\} ; \quad a_{j k}=\left(\frac{\partial \phi_{1 k}}{\partial n}, \phi_{2 j}\right) ; \quad k, i=\overline{1, N} ; j=\overline{1, M} ; \\
P=\left\{p_{k i}\right\} ; \quad p_{k i}=\left(\phi_{1 k}, w_{i}\right) ;, B=\left\{b_{j k}\right\} ; \quad b_{j k}=\left(\phi_{2 j}, w_{k}\right),
\end{gathered}
$$

we come to the next eigenvalue problem:

$$
\omega^{2} E C+H_{\omega} C+\omega^{2} \rho_{l} P C+\omega^{2} \rho_{l} B D=0 ; \omega^{2} E D+g A C+H_{\chi} D=0 .
$$

Introduce also for simplicity vectors and matrixes of doubled dimension $N+M$

$$
X=\left(\begin{array}{l}
C \\
D
\end{array}\right) ; H=\left(\begin{array}{cc}
E+\rho_{2} P & \rho_{2} B \\
0 & E
\end{array}\right) ; G=\left(\begin{array}{cc}
H_{\omega} & 0 \\
g A & H_{\chi}
\end{array}\right) .
$$

It brings us to the following eigenvalue problem

$$
\left(G-\omega^{2} H\right) X=0 \text {. }
$$

So the free vibration analysis of an elastic shell coupled with liquid sloshing is reduced to the solution of eigenvalue problem (18). It would be noted that hereinbefore we did not assume that the shell considered was a shell of revolution only. Hereinafter we will use the finite element method to define basic functions $w_{j}$, and the boundary element method to define basic functions $\varphi_{1 k}, \varphi_{2 k}$. 


\section{Systems of the boundary integral equations and some remarks about their numerical implementation}

Now and hereinafter we consider the shells of revolution only. The basic procedure is to start with the standard boundary integral equation for the surface potential [5], replace the Cartesian co-ordinates $(x, y, z)$ with cylindrical coordinates $(r, \theta, z)$, and integrate with respect to $z$ and $\theta$. We use furthermore the cylindrical coordinate system and represent unknown functions as Fourier series by the circumferential coordinate

$$
w_{k}(r, z, \theta)=w_{k}(r, z) \cos \alpha \theta ; \quad \phi_{i j}(r, z, \theta)=\phi_{i j}(r, z) \cos \alpha \theta ; \quad i=1,2,
$$

where $\alpha$ is a given integer (the number of nodal diameters).

We will seek both harmonic functions $\varphi_{1 k}$ and $\varphi_{2 k}$. as the sums of potentials of single and double layers [5], i.e. we will use the direct boundary element method formulation. Hereinafter we will drop indexes $i j$ for simplicity. It is assumed here that $S=S_{1} \cup S_{0}$ and the points $P$ and $P_{0}$ belong to the surface $S$. The value $\left|P-P_{0}\right|$ represents the Cartesian distance between the points $P$ and $P_{0}$. Let $\Gamma$ be a generator of the surface $S_{1}$. We obtain the following system of singular integral equations for unknown functions $\varphi$ and $q$ :

$$
\begin{array}{r}
2 \pi \phi\left(z_{0}\right)+\int_{\Gamma} \phi(z) Q\left(z, z_{0}\right) r(z) d \Gamma-\int_{0}^{R} q(\rho) \Psi\left(P, P_{0}\right) \rho d \rho=\int_{\Gamma} w(z) \Psi\left(P, P_{0}\right) r(z) d \Gamma_{1} ; P_{0} \in S_{1} ; \\
\int_{\Gamma} \phi(z) Q\left(z, z_{0}\right) r(z) d \Gamma-\int_{0}^{R} q(\rho) \Psi\left(P, P_{0}\right) \rho d \rho=\int_{\Gamma} w(z) \Psi\left(P, P_{0}\right) r(z) d \Gamma_{1} ; P_{0} \in S_{0} .
\end{array}
$$

Here

$$
\begin{gathered}
Q\left(z, z_{0}\right)=\frac{4}{\sqrt{a+b}}\left\{\frac{1}{2 r}\left[\frac{r^{2}-r_{0}^{2}+\left(z_{0}-z\right)^{2}}{a-b} \mathrm{E}_{\alpha}(k)-\mathrm{F}_{\alpha}(k)\right] n_{r}+\frac{z_{0}-z}{a-b} \mathrm{E}_{\alpha}(k) n_{z}\right\} ; \\
\Psi\left(P, P_{0}\right)=\frac{4}{\sqrt{a+b}} \mathrm{~F}_{\alpha}(k) ; \mathrm{E}_{\alpha}(k)=(-1)^{\alpha}\left(1-4 \alpha^{2}\right) \int_{0}^{\pi / 2} \cos 2 \alpha \psi \sqrt{1-k^{2} \sin ^{2} \psi} d \psi ; \\
\mathrm{F}_{\alpha}(k)=(-1)^{\alpha} \int_{0}^{\pi / 2} \frac{\cos 2 \alpha \psi d \psi}{\sqrt{1-k^{2} \sin ^{2} \psi}} ; a=\rho^{2}+\rho_{0}^{2}+\left(z^{*}-z_{0}\right)^{2} ; \quad b=2 \rho \rho_{0} ; k^{2}=\frac{2 b}{a+b}
\end{gathered}
$$

In doing so, the function $\varphi$, defined on the surface $S_{1}$, presents the pressure on the moistened shell surface and the function $q$, defined on the surface $S_{0}$, is the flux. To define potentials $\varphi_{2 k}$ we use equation (20) and introduce next integral operators:

$$
\begin{gathered}
A \psi_{1}=2 \pi \psi_{1}+\iint_{S_{1}} \psi_{1} \frac{\partial}{\partial n} \frac{1}{r\left(P, P_{0}\right)} d S_{1} ; B \psi_{0}=\iint_{S_{0}} \psi_{0} \frac{1}{r} d S_{0} ; \\
C \psi_{0}=\iint_{S_{0}} \psi_{0} \frac{\partial}{\partial z}\left(\frac{1}{r}\right) d S_{0} ; D \psi_{1}=-\iint_{S_{1}} \psi_{1} \frac{\partial}{\partial n} \frac{1}{\left|P-P_{0}\right|} d S_{1} ; F \psi_{0}=\iint_{S_{0}} \psi_{0} \frac{1}{r} d S_{0} .
\end{gathered}
$$

Then the boundary value problem (8), (10) takes the form

$$
A \psi_{1}=\left(\chi^{2} / g\right) B \psi_{0}-C \psi_{0} ; P_{0} \in S_{1} ; D \psi_{1}=2 \pi E \psi_{0}-\left(\chi^{2} / g\right) F \psi_{0} ; P_{0} \in S_{0} \text {. }
$$

After excluding function $\psi 1$ from these relations, we will obtain the 
eigenvalue problem and its solution gives natural modes and frequencies of liquid sloshing in the rigid tank

$$
\left(D A^{-1} C+E\right) \psi_{0}-\lambda\left(D A^{-1} B+F\right) \psi_{0}=0 ; \quad \lambda=\chi^{2} / g .
$$

Numerical solution of integral equation system (20) and evaluation of integral operators (21) were obtained by the BEM with a constant approximation of unknowns $\varphi$ and $q$ inside elements. It would be noted that internal integrals in (20), (21) are complete elliptic integrals of first and second kinds. As the first kind elliptic integrals are non-singular, one can successfully use standard Gaussian quadratures for their numerical evaluation. For second kind elliptic integrals we have applied here the approach based on the following characteristic property of the arithmetic geometric mean $\operatorname{AGM}(a, b)$ (see [12]):

$$
\int_{0}^{\pi / 2} \frac{d \theta}{\sqrt{a^{2} \cos ^{2} \theta+b^{2} \sin ^{2} \theta}}=\frac{\pi}{2 A G M(a, b)} .
$$

To define $\operatorname{AGM}(a, b)$ there exist the simple Gaussian algorithm, described below,

$$
\begin{gathered}
a_{0}=a ; b_{0}=b ; a_{1}=\frac{a_{0}+b_{0}}{2} ; b_{1}=\sqrt{a_{0} b_{0}} ; \ldots a_{n+1}=\frac{a_{n}+b_{n}}{2} ; b_{n+1}=\sqrt{a_{n} b_{n}} ; \ldots \\
A G M(a, b)=\lim _{n \rightarrow \infty} a_{n}=\lim _{n \rightarrow \infty} b_{n} .
\end{gathered}
$$

It is a very effective method to evaluate the elliptic integrals of the second kind. For instance, convergence $\varepsilon=\left|a_{n}-b_{n}\right|<10^{-8}$ was achieved after 6 iterations.

So we have the effective numerical procedures for evaluation of inner integrals, but integral equations (22), (23) involve external integrals with logarithmic singularities and thus the numerical treatment of these integrals will also have to take into account the presence of this integrable singularity. Here integrands are distributed strongly non-uniformly over the element and standard integration quadratures fail in accuracy. So we treat these integrals numerically by special Gauss quadratures $[5,13]$ and apply the technique proposed in [14].

\section{Some numerical results}

The study of free vibration characteristics of the elastic cylindrical shell interacting with a liquid is presented here. It is supposed that $\alpha=0,1$ in equations (19)-(21), i.e. we consider both axisymmetric and non- axisymmetric modes.

Three systems of basic functions have been built. The modes of the empty shell are the first one. The second system consists of free vibration modes of the elastic shell without including the force of gravity, and the third one represents modes of liquid sloshing of the rigid tank with the gravity force.

Consider the circular cylindrical shell with a flat bottom and having the following parameters: the radius is $R=1 \mathrm{~m}$, the thickness is $h=0.01 \mathrm{~m}$, the length $L=2 \mathrm{~m}$, Young's modulus $E=2 \cdot 10^{5} \mathrm{MPa}$, Poisson's ratio $v=0.3$, the material's density is $\rho=7800 \mathrm{~kg} / \mathrm{m}^{3}$, the fluid density $\rho_{1}=1000 \mathrm{~kg} / \mathrm{m}^{3}$. The fluid filling level is denoted as $H$. The shell is assumed to be pin-connected over its contour and boundary conditions are following: $u_{r}=u_{z}=u_{\theta}=0$ to $z=0$ and 
$r=R$. The own modes of the empty shell vibrations were obtained using the finite element method (FEM) as it was described in [8]. The second set of basic functions was obtained in $[7,8]$. To obtain the third set we consider liquid sloshing in the rigid cylindrical shell. We use the analytical solution [11] that can be expressed in the next form for testifying the proposed numerical algorithm:

$$
\frac{\chi_{k}^{2}}{g}=\mu_{k} \tanh \left(\mu_{k} \frac{H}{R}\right), k=1,2, . . ; \phi_{2 k}=J_{\alpha}\left(\frac{\mu_{k}}{R} r\right) \cosh \left(\frac{\mu_{k}}{R} z\right) \cosh ^{-1}\left(\frac{\mu_{k}}{R} H\right) .
$$

Noted that in (23) values $\mu_{k}$ are roots of the equation $J_{\alpha}^{\prime}(x)=0$, where $J_{\alpha}(x)$ is Bessel function of the first kind, $\chi_{k}, \varphi_{2 k}$ are frequencies and modes of liquid sloshing in the rigid cylindrical shell. The numerical solution was obtained by using the BEM as it was described beforehand. In present numerical simulation we used 60 boundary elements along the bottom, 60 elements along wetted cylindrical parts and 100 elements along the radius of free surface. Figure 2 shows the first three modes of liquid sloshing in the rigid cylindrical shell for $\alpha=0$ and $\alpha=1$.

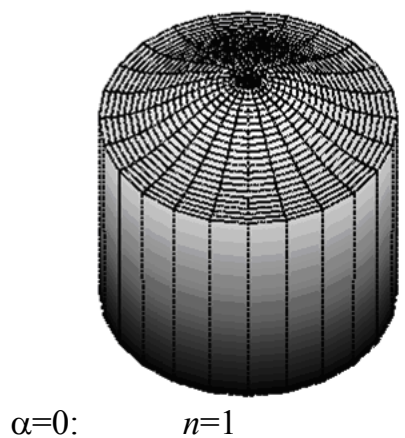

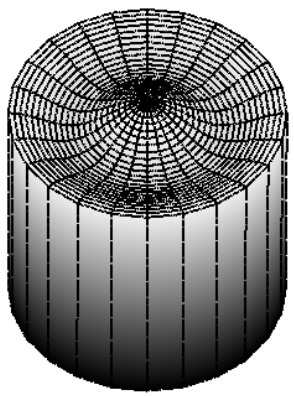

$n=2$

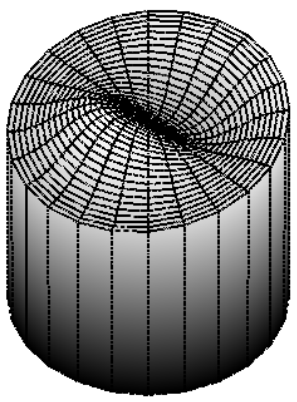

$n=2$

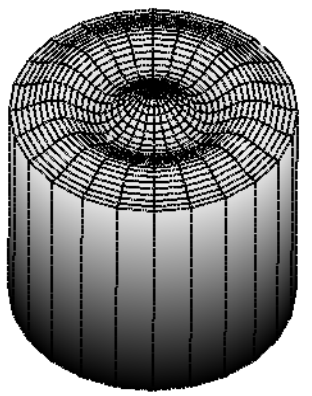

$n=3$

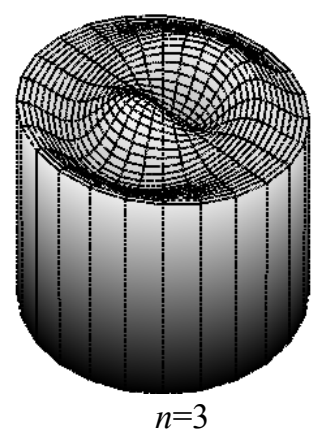

Figure 2: Modes of liquid sloshing in the rigid cylindrical shell.

Table 1 below provides the numerical values of the natural frequencies of liquid sloshing for nodal diameters $\alpha=0$ and $\alpha=1$. The obtained numerical results are compared with those received using formulae (23). 
Table 1: Comparison of analytical and numerical results.

\begin{tabular}{|c|c|c|c|c|c|c|}
\hline \multicolumn{2}{|c|}{} & $n=1$ & $n=2$ & $n=3$ & $n=4$ & $n=5$ \\
\hline \multirow{2}{*}{$\alpha=0$} & BEM & 3.815 & 7.019 & 10.180 & 13.333 & 16.480 \\
\cline { 2 - 7 } & $(23)$ & 3.815 & 7.016 & 10.173 & 13.324 & 16.470 \\
\hline \multirow{2}{*}{$\alpha=1$} & BEM & 1.657 & 5.332 & 8.540 & 11.711 & 14.889 \\
\cline { 2 - 7 } & $(23)$ & 1.657 & 5.329 & 8.536 & 11.706 & 14.863 \\
\hline
\end{tabular}

Figures 3 and 4 also demonstrate good agreement between numerical and analytical data.

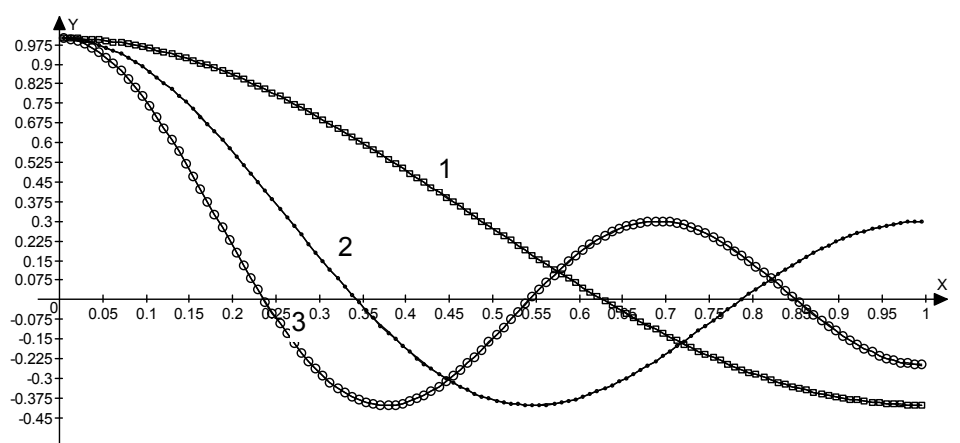

Figure 3: Numerically and analytically obtained modes.

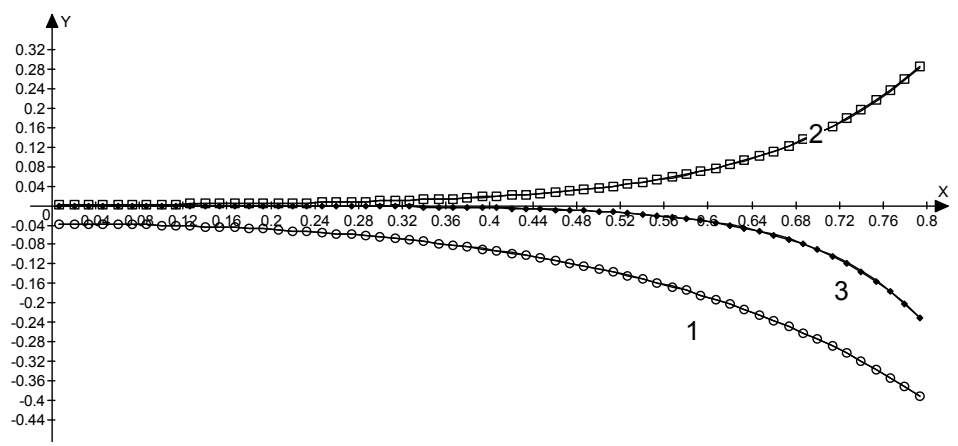

Figure 4: $\quad$ Sloshing modes on the vertical wall.

In Figure 3 the distributions of first three sloshing modes for $\alpha=0$ on the free surface are shown. The solid lines denote modes obtained by analytical expression (23) at $z=H$.

Figure 4 demonstrates the distributions of the same modes on the rigid vertical wall. The lines pointed with circles and squares denote numerical solutions. Numbers 1, 2, 3 correspond to the first, second and third modes of liquid sloshing. It would be noted that the accuracy $\varepsilon=10^{-4}$ has been achieved here. 
Functions $\varphi_{1 k}$ were calculated by the BEM based method developed in $[8$, 16].

Table 2 provides the numerical values of natural frequencies of vibration for empty and fluid-filled cylindrical tanks. Here coefficients $n_{S}, n_{L}$ indicate numbers of modes of the shell and liquid involved in coupled vibrations, $n$ is the number of the coupled mode. For numerical simulation we have used here 4 shell modes and 5 sloshing modes.

Table 2: Frequencies of empty and fluid-filled tanks.

\begin{tabular}{|c|c|c|c|c|c|c|c|c|}
\hline \multicolumn{5}{|c|}{$\alpha=0$} & \multicolumn{4}{|c|}{$\alpha=1$} \\
\hline$n$ & $n_{S}$ & $n_{L}$ & $\begin{array}{c}\text { Empty } \\
\text { elastic } \\
\text { tank }\end{array}$ & $\begin{array}{l}\text { Fluid- } \\
\text { filled } \\
\text { tank }\end{array}$ & $n_{S}$ & $n_{L}$ & $\begin{array}{c}\text { Empty } \\
\text { elastic } \\
\text { tank }\end{array}$ & $\begin{array}{l}\text { Fluid- } \\
\text { filled } \\
\text { tank }\end{array}$ \\
\hline 1 & & 1 & & 6.11932 & & 1 & & 4.03300 \\
\hline 2 & 1 & 1,2 & 23.233 & 7.94464 & & 2 & & 7.2328 \\
\hline 3 & & 2 & & 8.29916 & & 3 & & 9.15547 \\
\hline 4 & & 3 & & 9.99588 & & 4 & & 10.7233 \\
\hline 5 & & 4 & & 11.4410 & & 5 & & 12.0857 \\
\hline 6 & & 5 & & 12.7239 & 1,2 & & 48.5207 & 21.9555 \\
\hline 7 & 2,1 & & 91.1011 & 43.8628 & 2,1 & & 139.708 & 79.7191 \\
\hline 8 & 3,2 & & 205.252 & 119.627 & $3,2,1$ & & 232.443 & 178.422 \\
\hline 9 & $4,3,2$ & & 365.795 & 238.695 & 4,3 & & 277.303 & 210.007 \\
\hline
\end{tabular}

The numerical analysis demonstrates that the lowest frequency corresponds to liquid sloshing at $\alpha=1$. The frequencies of the fluid-filled shell are essentially lower than those of the empty shell. It also would be noted that there exist an interaction between both liquid and wall vibrations. It was observed for the first shell mode. This does not allow us to consider separately the spectra of frequencies of the shell and liquid sloshing.

\section{Conclusions}

The shell vibrations coupled with liquid sloshing under the force of gravity were considered. The free vibration analysis of the elastic cylindrical shell was carried out using the proposed techniques. We introduce the representation of the velocity potential as the sum of two potentials, one of them corresponds to problem of the fluid free vibrations in the rigid shell and another one corresponds to the problem of fluid-filled elastic shell vibrations without including the gravitational component. The spectrum of frequencies for cylindrical tank was analysed. The proposed method allows us to carry out numerical simulation for different value of gravitational acceleration $g$.

\section{Acknowledgement}

The authors gratefully acknowledge Professor Carlos Brebbia, Wessex Institute of Technology, for his constant support and interest in our research. 


\section{References}

[1] G. Popov, S. Sankar, T. S. Sankar, G. H. Vatisin, Liquid Sloshing in Rectangular Road Container. Computers \& Fluids, V. 21, N 4, pp. 551569, 1999.

[2] Dukowicz, J. K., Dvinsky, A. S., Approximate Factorization as a High Order Splitting for the Implicit Incompressible Flow Equations, $J$. Comput. Phys., 102, pp. 330-336, 1992.

[3] Tezduyar T. E. Finite Element Methods for Fluid Dynamics with Moving Boundaries and Interfaces. Encyclopedia of Computational Mechanics, (3): Fluids, pp. 1-55, 2004.

[4] Recent Advances in Boundary Element Methods A Volume to Honor Professor Dimitri Beskos Manolis, George; Polyzos, Demosthenes Eds., XXXVIII, 470 pp., 2009.

[5] Brebbia, C. A., Telles, J. C. F., Wrobel, L. C. Boundary Element Techniques, Springer-Verlag: Berlin and New York, 1984.

[6] J. Cappello, A. Sauret, F. Boulogne, E. Dressaire, H. Stone, Damping of liquid sloshing by foams: from everyday observations to liquid transport, Journal of Visualization, pp. 1-3, 2014.

[7] Strelnikova, E., Yeseleva, E., Gnitko, V., Naumenko, V. Free and forced vibrations of the shells of revolution interacting with the liquid. Proc. of XXXII Conference "Boundary elements and other mesh reduction methods" WITPress, Transaction on Modeling and Simulation, pp. 203$211,2010$.

[8] Ventsel, E., Naumenko, V., Strelnikova, E., Yeseleva, E. Free vibrations of shells of revolution filled with a fluid. Engineering analysis with boundary elements, 34, pp. 856-862, 2010.

[9] Gnitko, V., Marchenko, U., Naumenko, V., Strelnikova, E. Forced vibrations of tanks partially filled with the liquid under seismic load. Proc. of XXXIII Conference "Boundary elements and other mesh reduction methods" WITPress, Transaction on Modeling and Simulation, pp. 285296, 2011.

[10] Chen, Y. H., Hwang, W. S., Ko, C. H. Numerical simulation of the threedimensional sloshing problem by boundary element method. Journal of the Chinese Institute of Engineers, 23(3), pp. 321-330, 2000.

[11] Ibrahim, R. A. Liquid sloshing dynamics: theory and applications. Cambridge University Press, 957 pp., 2005.

[12] David A. Cox. The Arithmetic-Geometric Mean of Gauss. L'Enseignement Mathématique, t. 30, pp. 275-330, 1984.

[13] Stroud, A. H., Secrest, D. Gaussian Quadrature Formulas. Prentice-Hall, Englewood, NJ, Cliffs, 206 pp., 1966.

[14] Naumenko, V. V., Strelnikova, H. A. Singular integral accuracy of calculations in two-dimensional problems using boundary element methods. Engineering analysis with boundary elements. № 26, pp. 95-98, 2002. 